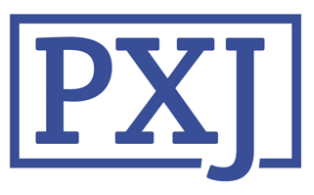

Patient Experience Journal

Volume 4

Issue 2 Special Issue: Patient Involvement

Article 7

2017

\title{
Increasing sustainability in co-design projects: A qualitative evaluation of a co-design programme in New Zealand
}

Lynne Margaret Maher Dr.

Ko Awatea

Brooke Hayward

Ko Awatea

Patricia Hayward

Counties Manukau Health

Chris Walsh Dr

Health, Quality \& Safety Commission

Follow this and additional works at: https://pxjournal.org/journal

Part of the Health and Medical Administration Commons, Leadership Studies Commons, Medical Humanities Commons, and the Organization Development Commons

\section{Recommended Citation}

Maher LM, Hayward B, Hayward P, Walsh C. Increasing sustainability in co-design projects: A qualitative evaluation of a co-design programme in New Zealand. Patient Experience Journal. 2017; 4(2):44-52. doi: 10.35680/2372-0247.1150.

This Research is brought to you for free and open access by Patient Experience Journal. It has been accepted for inclusion in Patient Experience Journal by an authorized editor of Patient Experience Journal. 


\title{
Increasing sustainability in co-design projects: A qualitative evaluation of a co-design programme in New Zealand \\ Dr. Lynne Margaret Maher, Ko Awatea, lynne.mahen@middlemore.co.nz \\ Brooke Hayward, Ko Awatea,brooke.hayward@middlemore.co.nz \\ Patricia Hayward, Counties Manukan Health, patricia.bayward@middlemore.co.n₹. \\ Dr. Chris Walsh, Health Quality \& SafetyCommission, chris.walsh@hqsc.govt.nz.
}

\begin{abstract}
The Health Quality \& Safety Commission New Zealand commissioned Ko Awatea, an innovation and improvement centre, to deliver a co-design programme to nine teams of healthcare providers. The co-design programme was part of Partners in Care, a broader programme developed in 2012 to support and enable patient engagement and participation across the health and disability sector. Teams received training, guidance and mentorship in Experience Based Design (EBD) methodology. ${ }^{1}$ We evaluated the co-design programme to explore barriers and facilitators to the sustainability of the co-design projects and the EBD approach. The evaluation involved seventeen semi-structured interviews with programme participants, including seven team members, five sponsors, four patients and the programme facilitator. A further two team members provided written feedback. Eight teams provided completed workbooks. Data from the interviews and workbooks was thematically analysed. Team members saw support from sponsors as important to increase visibility and successful completion of co-design projects, mitigate barriers, and to secure resources and buy-in from peers. Five of nine participating teams reported dissatisfaction with the support received. Communication and competing priorities were challenges to sponsor engagement. Sharing co-design skills with peers and alignment with organisational strategy were seen as important for sustainability. Teams identified lack of secured resources or staff time, and consumer or staff attrition as key barriers to sustainability. The conclusion: buy-in from sponsors and senior leaders, support from colleagues, user-friendliness of co-design tools, consumer and staff availability, alignment, and system or culture change were key factors that influenced project sustainability.
\end{abstract}

\section{Keywords}

Patient engagement, patient experience, Experience Based Design, qualitative methods, co-design, sustainability

\section{Background}

The experiences that patients, the public and healthcare staff have when they receive or deliver healthcare services can be used to improve care and transform services..$^{2,3}$

Experience Based Design (EBD), also known as codesign, is an evidence-based approach to designing better healthcare services that draws out and captures the experiences of patients, caregivers and healthcare staff for the purpose of improving services. This ensures that healthcare professionals understand experiences from the perspective of staff, patients and caregivers. ${ }^{1,3}$ The EBD approach entails the use of a specific process, which has been adapted for use in New Zealand (Figure 1).

The EBD approach has also been applied in healthcare services in England, Canada, the USA, Australia and New Zealand. ${ }^{1,5-8}$
Co-design approaches aim to understand and celebrate positive experiences and to identify and improve negative experiences. The process of improvement requires levels of organisational change. However, between 33 and 70 per cent of organisational change is not sustained.9-12

Literature suggests that the sustainability of change is influenced by process, staff and organisational factors. ${ }^{10,12}$ Process factors relate to real or perceived benefits beyond helping patients, credibility of evidence for the change, the adaptability of the improved process and the effectiveness of the system to monitor progress. Staff factors include staff involvement and training to sustain the process, staff behaviours towards sustaining change, and engagement by senior and clinical leaders. Organisational factors include whether the proposed improvements fit with the organisation's strategic aims and culture and the existence of infrastructure for sustaining change. ${ }^{10}$ 
Figure 1. Summary of project phases for the Partners in Care co-design projects ${ }^{4}$

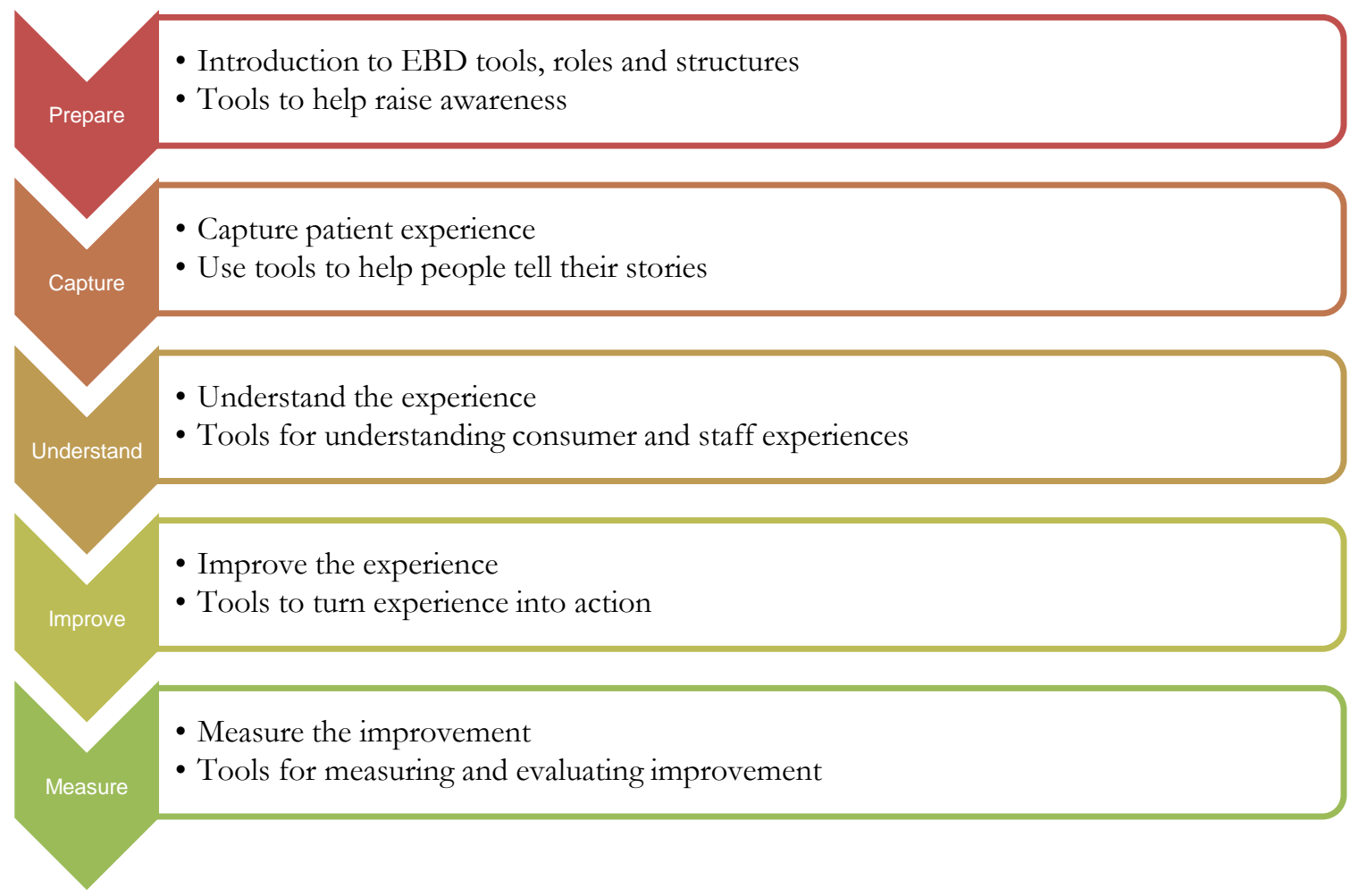

This article investigates barriers and facilitators to sustainability in co-design projects undertaken at nine healthcare organisations as part of the Health Quality \& Safety Commission New Zealand's Partners in Care codesign programme.

Partners in Care was originally developed in 2012 by the Health Quality \& Safety Commission New Zealand (HQSC), a crown entity which leads and coordinates health quality and safety activity in New Zealand, to support and enable patient engagement and participation across the health and disability sector in decision-making about their own health and the delivery of health and disability services. A co-design work stream has been a consistent part of Partners in Care.

HQSC commissioned Ko Awatea, the health system innovation and improvement centre at Counties Manukau Health $(\mathrm{CMH})$ in Auckland, to deliver the co-design element of Partners in Care for its third iteration from October 2014 to the end of April 2015. In this iteration, Ko Awatea worked with the nine healthcare organisations to deliver content to support the core principles of the programme:

- To achieve a partnership between patients staff and carers.
- An emphasis on experience rather than attitude or opinion.

- Narrative and storytelling approach to identify 'touch points'.

- An emphasis on the co-design of services.

- Systematic evaluation of improvements and benefits.

Programme participants used a systematic process to capture, understand and improve safety and other aspects of the care journey through the co-design of healthcare processes and services. The programme also contributes to vision and values assumed by many healthcare services to work in partnership with their communities to deliver patient-centred care.

An evaluation of the co-design programme aimed to:

- describe the challenges and solutions by participating teams to increase the engagement of patients to co-design of health services

- describe how the approach is being embedded into daily practice, and identify opportunities to increase sustainability of the approach

- determine the level of leadership support provided to team members and how this impacts on the achievements and learning experiences of teams 
- $\quad$ produce advice on how to engage patients in the co-design of health services.

This article focusses on the evaluation objectives that relate to leadership and sustainability of co-design projects and the EBD approach.

\section{Programme delivery}

Project teams from nine healthcare providers participated in the co-design programme. Collectively, this included 56 healthcare professionals and 17 patients. Patients engaged at one of two levels: those who contributed feedback, information and perspectives about their healthcare experiences to project teams; and those who actively participated in ongoing communication and decisionmaking with project teams, in addition to contributing feedback, information and perspectives.

Teams sought the leadership of a project sponsor, who was responsible for supporting project teams at each site. Depending on the needs of each team, the responsibilities of sponsors involved securing staff release time to dedicate to projects, socialising the projects with other senior leaders, assisting teams to problem solve and maintaining project momentum. The project sponsor was typically a member of staff who was known to the project team, had an interest in supporting co-design approach in their organisation, and was in a management or leadership position, such as a clinical leader, service manager, general manager, quality manager or director.

Participation in the co-design programme commenced with team members and consumers from each of the project teams attending one of two masterclasses. The masterclass aimed to increase participants' competencies in:

- understanding the context, value and evidence base for working closely with patients and their families

- awareness of a staged process to engage patients, capture their experiences of care, organise and identify themes for improvement and to codesign future services

- knowledge of a range of specific customer service design methods including observation, shadowing, interviewing, emotion mapping and co-design

- application of these methods to National Patient Safety Campaign work streams.

The masterclasses included a mix of presentations, group work, and discussion to maximise learning.

Following the masterclasses, Ko Awatea delivered a sevenmonth course to provide ongoing education in co-design methodology, mentorship and support to participating teams. The course comprised seven one-hour WebEx sessions, which incorporated formal teaching and opportunities for participating teams to share their progress and ask questions. In addition to the formal teaching delivered at the initial masterclass and subsequent WebEx sessions, participants were invited to contact Ko Awatea for further guidance as required. Guidance was provided through coaching calls and email.

During the programme, the facilitator tracked the progress of the projects to ensure appropriate progress was being made and that they were following the co-design methodology correctly. The tracking included project teams presenting aspects of their work during the WebEx sessions and completing workbooks twice during the programme period to capture progress and learning. The workbooks were reviewed twice by the Ko Awatea programme facilitator who provided detailed feedback and direction as teams progressed.

Workbooks captured:

- $\quad$ evidence of each project team's work and feedback from each phase of the co-design approach

- descriptions of how the team engaged leaders, staff colleagues and patients, including what worked well and any challenges

- practical experience of utilising tools and methods that increased the engagement of patients and led to co-design of health services

- $\quad$ stories/narratives that demonstrated the impact of working closely with patients

- the impact that participating in this programme had on team members, patients and other people they were working with, and the organisation they worked for.

Project teams also completed a case study template as they captured a 500 word case study describing each project. Programme participants had access to a wide range of resources and learning material through the HQSC website. This included relevant peer-reviewed papers, other helpful documents and website links about patient experience. Participants could also share their own learning resources and useful documents with other participants through this website.

\section{Evaluation}

The evaluation framework for the co-design programme was developed jointly by the Research and Evaluation team at Ko Awatea, the programme facilitator and the HQSC Partners in Care director. The evaluation applied qualitative data collection methods to gain in-depth information from key stakeholders to meet evaluation objectives. Data collection methods used were: 
- $\quad$ study and analysis of teams' workbooks, completed case study templates and presentations for contributions to WebEx sessions

- $\quad$ semi-structured interviews with team members and senior leader project sponsors (sponsors)

- $\quad$ semi-structured interviews with patients

- $\quad$ semi-structured interview with the programme facilitator.

Completed workbooks from each team were provided directly to the Research and Evaluation team by the programme facilitator with the consent of participants, twice throughout the programme period (January and June 2015).

The programme facilitator made initial contact by email with members of participating teams, sponsors and consumers. The purpose of the initial contact was to introduce the lead investigator, communicate evaluation objectives and answer queries potential participants typically have about the evaluation. The contact details for the lead investigator were also provided so that participants could make contact about any questions, concerns or complaints about the evaluation.

Following initial contact, all potential evaluation participants were provided with information detailing the evaluation objectives, participant requirements, risks, and use of data. This was accompanied by an invitation to participate in the evaluation. Those who did not respond received telephone or email reminders. Those who did respond were sent a short survey to assist with interview scheduling. Interviews were then confirmed by telephone.

Due to the location and preferences of evaluation participants, most participant interviews with sponsors, team members and consumers were conducted over the telephone. A face-to-face interview was held with the programme facilitator and one patient.

Questions in the interview schedules were grouped around themes (Table 1).

\section{Analysis}

A written record of each evaluation interview was sent to the interviewee for verification and to highlight any missed points. Interview records were then de-identified to protect the confidentiality of evaluation participants, and thematically analysed.

Workbook materials were filtered for relevance to evaluation questions and thematically analysed alongside interview data.

\section{Findings}

In total, 17 semi-structured interviews were conducted, involving seven team members, five sponsors, four patients, and the programme facilitator. A further two team members provided feedback in written form. Completed workbooks were obtained for eight of the nine healthcare organisations participating in the co-design programme. Due to staff turnover, one healthcare service was unable to complete the final workbook.

\section{Table 1. Question themes in interview schedules for stakeholder groups}

\begin{tabular}{|c|c|}
\hline Stakeholder & Themes \\
\hline Team members & $\begin{array}{ll}\text { - } & \text { Staff experiences of approaching patients } \\
\text { - } & \text { Securing participation from patients } \\
\text { - } & \text { Guide for approaching patients } \\
\text { - } & \text { Learning and sustainability } \\
\text { - } & \text { Support from sponsors } \\
\text { - } & \text { Opportunities for improvement }\end{array}$ \\
\hline Sponsors & $\begin{array}{ll}\text { - } & \text { Support offered } \\
\text { - } & \text { Learning and sustainability } \\
\text { - } & \text { Opportunities for improvement } \\
\end{array}$ \\
\hline Patients & 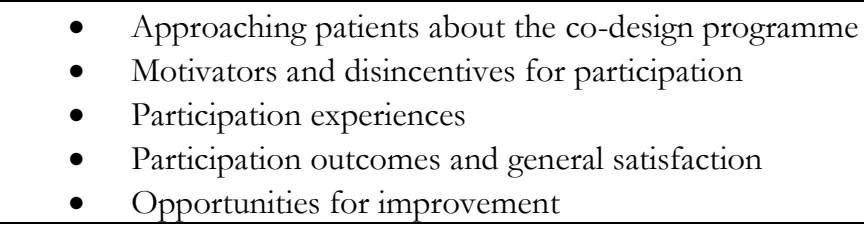 \\
\hline Programme facilitator & $\begin{array}{ll}\text { - } & \text { Staff experiences approaching patients } \\
\text { - } & \text { Learning and sustainability } \\
\text { - } & \text { Support } \\
\text { - } & \text { Opportunities for improvement } \\
\end{array}$ \\
\hline
\end{tabular}


Ideas from sponsors and team members for increasing sustainability are summarised in Table 2 .

\section{Buy-in from sponsors and senior leaders}

A key theme in the support needs identified by team members was buy-in and engagement from sponsors and other senior leaders. Five of the nine participating teams reported some dissatisfaction with buy-in or engagement received from sponsors or other senior leaders, which impacted project progress:

\section{"Co-design is new to the [organisation] and, whilst the senior management team is supportive, there is lack of understanding of the value for our services and therefore capacity to carry out the project is limited due to competing priorities."}

In particular, team members indicated the need for more support around mitigating barriers to solution implementation and socialising the importance of the approach with other staff. A barrier to sustainability of the co-design approach is that envisioned changes often sit beyond the capacity, authority and scope of project teams. Buy-in from senior leadership and other staff was seen as pivotal to mitigate barriers and implement solutions:

"It is difficult to make changes that are beyond your
control, authority or leadership. There were several people
involved in this project who impacted outcomes, or had to
provide consent or approval for changes, as well as
international standards to consider."

"A key challenge of the co-design approach was that a select group of staff worked on improvement, but instigating changes required the collaboration of a much bigger group of staff, from frontline to management... We significantly underestimated how extensive the communications should be."

Lack of secured resources to implement solutions or dedicate staff time to projects was also identified as a key barrier to project sustainability and achieving outcomes.

Team members described buy-in and engagement in the following ways:

- understanding the co-design approach and socialising it with other staff at all levels of the organisation

- $\quad$ securing resources such as staff release time and funding for solutions/system change initiatives

- $\quad$ problem-solving with project teams when they encountered barriers

- $\quad$ being directly involved in some project meetings and WebEx sessions

- $\quad$ contributing to discussion, planning and patient engagement at operational and strategic levels

- helping to establish ongoing organisational development in co-design approaches.

The programme facilitator emphasised the shared responsibility of sponsors and team members to maintain open channels of communication about the level of support needed. One team member commented that, "It was a little unclear what the role of the sponsor was", which

Table 2. Summary of ideas from team members and sponsors to increase sustainability of the EBD approach

\begin{tabular}{l}
\hline $\begin{array}{l}\text { Promoting the projects and increasing visibility of the work and patient voices within healthcare services to support } \\
\text { cultural change around consumer voices. }\end{array}$ \\
\hline $\begin{array}{l}\text { Disseminating skills more widely across healthcare systems by creating opportunities for experiential learning, or } \\
\text { learning through observation with new co-design projects. }\end{array}$ \\
\hline Building 'people power' through engagement with students, volunteers or others who could be involved in projects. \\
\hline $\begin{array}{l}\text { Increasing buy-in from sponsors or other senior leaders to enable or endorse (i) patients engagement approaches, and } \\
\text { (ii) changes recommended by project teams. }\end{array}$ \\
\hline $\begin{array}{l}\text { Involving more patients and team members in project teams to maintain momentum and mitigate staff turnover and } \\
\text { consumer attrition from projects. }\end{array}$ \\
\hline $\begin{array}{l}\text { Continue building relationships with patients and other health professionals to share the co-design approach with an } \\
\text { opportunity-based, rather than a fear-based, response. }\end{array}$ \\
\hline Align projects with broader/wider projects or strategic directions of healthcare services. \\
\hline Embed the approach within policy, procedure or other system changes. \\
\hline $\begin{array}{l}\text { Embed the approach in existing training and development opportunities that are already funded for patients and } \\
\text { healthcare professionals. }\end{array}$ \\
\hline $\begin{array}{l}\text { Seek funding opportunities to secure time of clinical staff to contribute to quality projects around patient stories/patient } \\
\text { voices. }\end{array}$ \\
\hline Dedicate adequate resources for funding of interventions. \\
\hline $\begin{array}{l}\text { Increase staff and leadership engagement in the masterclass training for increased buy-in and understanding of the value } \\
\text { of patient voices. }\end{array}$ \\
\hline
\end{tabular}


resulted in some reluctance to approach the sponsor for support when barriers were encountered. Conversely, some sponsors acknowledged that they could have been more proactive in following up with team members to see if any support was required.

Two sponsors suggested scheduling structured support and project management time to ensure time was dedicated to the project and not "eaten up juggling other priorities". Finding time among competing priorities was the biggest challenge for sponsors in supporting project teams: "It becomes another job in all the jobs you bave to do".

Patients and team members identified key attributes of effectiveness for sponsors supporting project teams (Table 3).

\section{Support from colleagues}

The understanding, willingness and energy of staff were a great support to project teams:

"The progress of the projects relies heavily on the enthusiasm and creativity of medical and nursing staff and [other health professionals] going the extra mile."

Team members identified the following opportunities to increase buy-in and engagement from their peers: (i) have all team members attend the masterclass; (ii) demonstrate the value of patient voices in real life examples; (iii) give staff an opportunity to see the approach in practice.

All team members and sponsors relayed the importance of sharing their new skills with others to increase sustainability of the co-design approach. When discussing how the approach has been shared with others, all team members referred to promoting project progress through communication channels. These channels included: team or clinical governance meeting updates; staff email updates; staff newsletters; promotional posters or photoboards; dissemination of project materials; District Health
Board Quality Awards applications; accreditation processes; and consumers sharing experiences at clinical governance board level. Team members saw these communications as integral to stimulating interest, gaining support and increasing awareness of co-design. However, beyond the promotional avenues described above, skill sharing did not occur through structured dissemination or training. Team members identified observation and experiential learning, or application of the approach in other projects, as preferred approaches for sharing skills:

"It feeds and grows by having other people observe it." "... bring more people on the journey by showing them the tools in practice."

\section{User-friendliness of co-design tools}

Five team members reported that co-design tools and resources provided through the co-design programme were being applied to new projects. In particular, materials from the masterclass training and WebEx sessions, the workbook structure, ' 5 whys' tool, programme facilitator support, patient experience capture tools and visual display tools, such as experience mapping, were raised as useful. Team members indicated continued use of the tools in new projects is enabled by having tools that:

- $\quad$ are easily adapted for localised use in other healthcare services, specialist areas or patient groups

- $\quad$ provide examples of use

- can be easily understood and used by patients (and in some instances re-designed in partnership with patients).

The ongoing availability of most of the tools, independent of funding or delivery of the programme, was also cited as a reason why they were useful.

\section{Consumer and staff availability}

Project momentum could be disrupted by patient attrition or staff turnover. Patients were sometimes away, had other

\section{Table 3. Attributes of effective sponsors/senior leaders for co-design project teams}

\begin{tabular}{|l|l|}
\hline Summary & Description \\
\hline Power & $\begin{array}{l}\text { In a position of influence to provide an authorising environment for staff time } \\
\text { committed to projects, to support proposed interventions/changes to systems or } \\
\text { services, and establish ongoing organisational development in co-design } \\
\text { approaches. }\end{array}$ \\
\hline People & Existing relationships and network knowledge of the relevant healthcare system. \\
\hline Passion & $\begin{array}{l}\text { Passionate and energetic about the co-design approach; a vested interest in the } \\
\text { project. }\end{array}$ \\
\hline Presence & $\begin{array}{l}\text { Availability to meet regularly with the project team to maintain engagement and } \\
\text { visibility and to show support; proactive in checking on progress; approachable for } \\
\text { direction and advice when needed. }\end{array}$ \\
\hline Problem solver & $\begin{array}{l}\text { Engage with project teams to find solutions to barriers encountered throughout } \\
\text { project phases. }\end{array}$ \\
\hline
\end{tabular}


commitments, or became too unwell to participate. This was a particular problem for project teams working with only one core patient. Likewise, when staff turnover occurred, project work could be reassigned to other staff members, which disrupted relationships with both patients and staff, and reduced project momentum.

\section{"It is more difficult for other staff to maintain the special connection with [the healthcare service] that the patient and their family have developed [with a specific member of staff]."}

\section{System or culture change}

Throughout the interviews, there were countless examples of how systems or culture change supported the sustainability of co-design projects.

Some of the system changes implemented as part of the co-design projects demanded a commitment to sustained use of co-design methods and a change in clinical practice. For example, one sponsor described changes in the handover process to allow direct input from the patient.

Several team members described the establishment of a patient council, or increased engagement with the existing patient council, at their organisation. They believed this to be a system change providing new pathways of engagement with patients that had occurred as a result of this iteration of the co-design programme.

Conversely, a lack of systemic or cultural change could threaten sustainability of the co-design projects. For example, one evaluation participant identified systemic or infrastructural issues, such as IT platforms and lack of integration between primary and secondary care, as key issues threatening the sustainability of the team's project.

Cultural changes identified included where team members or sponsors observed a change in the behaviour or attitudes of clinical staff. For example, one team member reported that staff were more person-centred and took a more collaborative approach with consumers than previously, visiting them and asking for their perspectives more often. Another commented, "Four years ago no one talked about having patients involved."

Conversely, the experience of one sponsor demonstrated that fear of change is a very real cultural barrier for some healthcare professionals.

\begin{abstract}
Alignment
Alignment was seen as a factor in sustainability. Sponsors suggested that aligning projects to policies or broader projects supports sustainability. Both team members and patients commented on the competing priorities in healthcare systems that can divert time and resources away from co-design projects, and how this impacted on the ability of team members to dedicate their time to the codesign project. Alignment with broader projects or policies would also assist when engagement from sponsors or senior leaders was complicated by conflicting agendas, as was the experience of one team member.

Other suggestions for alignment were embedding codesign in written policy, procedural standards, training or evaluation methodology.
\end{abstract}

\section{Discussion}

The evaluation objectives relating to leadership and sustainability focussed on: (i) identifying opportunities to increase sustainability of the approach; (ii) determining the level of support sponsors provided to team members; (iii) how the level of support from sponsors impacted on the achievements and learning experiences of teams.

Increased sustainability relied heavily on the engagement of sponsors in senior leadership positions. Project teams needed the support of sponsors to manage challenges and share successes. To achieve this, project teams and sponsors would have benefited from a clear shared understanding of their individual roles and of how they would work together. In the co-design projects, lack of clarity among team members about the sponsor's role inhibited communication. Project teams needed the

\section{Box 1. Key learning}

- Establish a clear understanding of roles and how best to work together between the project team and the sponsor from the outset.

- Maintain open communication channels about support needs and project progress to keep sponsors engaged.

- Align projects with organisational priorities and goals to engage sponsors and achieve long-term sustainability.

- Engage with more than one patient on the project team to mitigate any risks associated with patient attrition.

- Ensure more than one staff member builds a rapport with patients to mitigate any risks associated with staff turnover. 
sponsor to be involved and visible in their support of the project. This required regular contact with the project team, as maintaining open communication channels about support needs and project progress was critical to maintain engagement by sponsors. Time pressures and competing demands on sponsors contributed to the lack of support afforded to teams. Consideration of sponsors' preferred form of communication, working patterns and deadlines, advance planning and early notice of meetings and support expectations would help project teams to secure and retain sponsor engagement. ${ }^{10}$

Our findings reinforce advice in the NHS Institute for Innovation and Improvement's Sustainability Model that project alignment with organisational priorities and with sponsors' current work objectives and issues facilitates sponsor engagement. ${ }^{10}$ Alignment with organisational strategy, goals and vision helps to ensure long-term success for improvement projects. ${ }^{10}$ When this alignment is communicated clearly, projects are more likely to secure crucial support from senior leaders. ${ }^{10}$

Team members felt that they did not receive enough support from sponsors or other senior leaders to make the most out of their participation in the programme. Buy-in and engagement from sponsors was fundamental to project teams being able to secure release time to work on their project, validate the co-design approach, overcome barriers to change, and implement recommended solutions. Lack of buy-in or engagement resulted in loss of project momentum, as team members continually needed to advocate about the value of patient voices in redesigning healthcare systems.

Bak et al. describe the importance of promoting the success of EBD work. ${ }^{13}$ All team members in our evaluation spoke about promoting project progress to stimulate interest and awareness of the co-design approach. Typically, skill sharing also occurred through the conceptualisation of new projects involving more staff, and day-to-day interactions between staff and patients in which other staff observe the application of new skills. This evaluation has highlighted the need for more structured or formalised training opportunities.

The user-friendliness of co-design tools and methods, and their adaptability to local context, encouraged their ongoing use within the core project and take-up in other projects. Tools provided through the co-design programme have been applied to new projects in many of the organisations that participated in the programme. Early plans for skills transfer could support sustainability by increasing the number of staff who understand the tools and methods of co-design and feel confident about using them.
We found that patient attrition and staff turnover disrupted project momentum for some teams. Patient availability needs to be considered early. The likelihood that patients may not be able to continue their input should be anticipated. Therefore, it is wise to engage with a number of patients from the start and plan ongoing recruitment. Similarly, the possibility of staff turnover during the project should be anticipated to mitigate its impact. One way teams can do this is by encouraging more than one staff member to build a rapport with the patients involved in the project.

Culture and systems change were identified as cornerstones of increased sustainability of co-design improvement approaches. Comments from team members and sponsors about observing a shift towards a more patient-centred, collaborative approach to care by staff reinforce findings by Iedema et al. ${ }^{8}$

\section{Limitations}

Due to the strictly voluntary nature of participation in this evaluation and previously noted patient attrition from projects, only a small group participated in this evaluation, including four of 17 patients (24 per cent) and 12 of 56 healthcare professionals (21 per cent). While there is a lot to learn from these participants, it is not possible to ascertain to what extent particular experiences may be similar or relevant to those of others. Having clinical staff participate in evaluation activities was difficult given their limited time capacity during working hours. Interview times were offered as early as $7 \mathrm{am}$, and as late as $8 \mathrm{pm}$ to facilitate participation.

The evaluation had a qualitative focus and was therefore able to capture in-depth the experiences of sponsors, team members and patients participating in the programme. It did not, however, involve the collection or analysis of quantitative data, and therefore rigour is more difficult to maintain, assess, and demonstrate.

\section{Conclusion}

Lack of sustainability in improvement and change management projects is a known risk. ${ }^{10-12}$ Support and buy-in from sponsors is an important factor to consider. A mutual understanding by sponsors and project leads of their roles, and an effective plan for ongoing communication, is essential. The ability to align co-design clearly with organisational strategy can strengthen links to sponsors and other senior leaders, thus increasing the importance of co-design within their work and development plans. This alignment also impacts positively on system or culture change. Staff need time and support from colleagues to undertake any co-design projects; sponsors have a pivotal role in enabling this. 


\section{References}

1. NHS Institute for Innovation and Improvement. The EBD approach - Using patient and staff experience to redesign better healthcare service. Coventry, UK: Author; 2009.

2. Boyd H, McKernon S, Mullin B, Old A. Improving healthcare through the use of co-design. $N Z$ Med J. 2012; 125(1357): 76-87.

3. Doyle C, Lennox L, Bell D. A systematic review of evidence on the link between patient experience and clinical safety and effectiveness. BMJ Open. 2013 Jan 3; 3(1): pii: e001570. doi: 10.1136/bmjopen-2012001570

4. Adapted from: NHS Institute for Innovation and Improvement. The EBD approach - Using patient and staff experience to redesign better healthcare service. Coventry, UK: Author; 2009. p. 17.

5. Tan L, Szebeko D. 2009. Co-designing for dementia: The Alzheimer 100 project. AMJ. 2009; 1(12): 185198. doi 10.4066/AMJ.2009.97

6. The EBD approach case studies. NHS Institute for Innovation and Improvement web site. http://www.institute.nhs.uk/quality and value/exper ienced based design/case studies.html Accessed January 26, 2016.

7. Co-Design Partners in Care. Health Quality \& Safety Commission New Zealand web site. http://www.hqsc.govt.nz/our-programmes/partnersin-care/work-streams/co-design-partners-in-care/ Updated November 19, 2015. Accessed January 26, 2016.

8. Iedema R, Eamon M, Piper D, et al. Codesigning as a discursive practice in emergency health services: The architecture of deliberation. I of Appl Behavioral Science. 2010; 46(1): 73-91. doi: 10.1177/0021886309357544

9. Fleiszer AR, Semenic SE, Ritchie JA, Richer M-C, Denis J-L. The sustainability of healthcare innovations: a concept analysis. J Adv Nurs. 2015; 71(7): 1484-1498.

10. NHS Institute for Innovation and Improvement. Sustainability Model. Coventry, UK: Author; 2009.

11. Beer M, Nohria N. Cracking the code of change. Harvard Business Review. 2000; 78: 133-141, 216.

12. Ford JH, Krahn D, Wise M, Anderson Oliver K. Measuring sustainability within the Veterans Administration Mental Health System Redesign Initiative. Q Manage Health Care. 2011; 20(4): 263-279.

13. Bak K, Macdougall L, Green E, Moody L, Obarski G. Hindsight is 20/20: Lessons learned after implementing experience based design. Patient Experience Journal. 2014: 1(2): Article 5. Available from: http://pxjournal.org/journal/vol1.iss $2 / 5$ 